\title{
Immediate and Long-Term Results of Transcatheter Closure of Patent Ductus Arteriosus-Comparison of Two Decades before and after Change in Antibiotic Infective Endocarditis Prophylaxis Guidelines
}

\author{
Annina Dietrich ${ }^{1,2}$, Daniel Quandt ${ }^{1,2}$, Oliver Kretschmar ${ }^{1,2}$ and Walter Knirsch ${ }^{1,2, *}$ \\ ${ }^{1}$ Pediatric Cardiology, Pediatric Heart Center, Department of Surgery and Children's Research Center, University Children's Hospital \\ Zurich, Zurich, Switzerland \\ ${ }^{2}$ University of Zurich, Zurich, Switzerland \\ *Corresponding Author: Walter Knirsch. Email: walter.knirsch@kispi.uzh.ch
}

Received: 24 April 2021 Accepted: 10 June 2021

\begin{abstract}
Objectives: To determine immediate and long-term follow-up of transcatheter closure of patent ductus arteriosus (PDA) in children. Background: National antibiotic prophylaxis (AP) guideline for infective endocarditis changed after 2009, the effect on practice of PDA closure is unknown. Methods: Observational single center study analyzing follow-up of PDA closure comparing two time periods before (2002-2009) and after (2010-2019) changes in AP guideline. Results: 332 patients (68.1\% female), median (interquartile range) age 3.0 years (1.5-5.7) and body weight $14.0 \mathrm{~kg}(10.0-19.3)$, were enrolled. PDA morphology was conical type A (50.3\%), window type B (1.2\%), tubular type C (40.1\%), complex type D (2.1\%), elongated type E (0.9\%) and other (5.4\%). Minimal PDA diameter and length were $1.9 \mathrm{~mm}(1.3-2.5)$ and $8.0 \mathrm{~mm}$ (6.2-10.2). PDA was closed using coils (56.3\%), Amplatzer Duct Occluders (41.9\%) and others (1.8\%). Complete closure rate was $61.1 \%$ at catheter intervention, $72.3 \%$ on day $1,87.7 \%$ after 6 months and $98.4 \%$ at last follow-up on echocardiography. Moderate complication rate (severity level 3) was $4.2 \%$ and major complication rate (severity level 4 ) $0.3 \%$, with no catastrophic complications (severity level 5). Annual PDA closure rate declined in the second time period (22.6/year vs. 15.5/ year, $p=0.018)$, PDA size increased $(1.6 \mathrm{~mm} v s .2 .0 \mathrm{~mm}, p=0.002)$ and proportion of coils decreased $(72.4 \% v s$. $37.1 \%, p<0.001)$. Conclusions: Interventional closure of PDA is associated with excellent closure rates during follow-up $(>98 \%)$ and only a small number of complications leading to reintervention or surgery. Change in AP guidelines changed indication for and practice of PDA closure.
\end{abstract}

\section{KEYWORDS}

Patent arterial duct; device closure; congenital heart disease; infective endocarditis

\section{Introduction}

Patent ductus arteriosus (PDA) is routinely closed by surgical ligation or catheter-based device procedure. Transcatheter PDA closure has been established as a safe and effective treatment and is currently the standard of care for PDA closure in children and adults. Even in small and preterm infants, interventional PDA closure has become more frequent [1]. Since the technical development of 
transcatheter PDA closure, new devices have continually been introduced, striving for better results, easier access, fewer complications and lower expenses [2].

In addition to the hemodynamic impact of the left-to-right shunt in patients with PDA, the risk of infective endocarditis (IE) is increased [3]. In the past, antibiotic IE prophylaxis (AP) was recommended in risk situations for transient bacteremia. Nowadays, prevention of IE focuses mainly on prevention measures such as good dental health. Swiss guidelines for prevention of IE have been adapted to those published by the American Heart Association in 2007 [4], focusing on non-pharmacological prevention rather than intermittent AP [5]. In addition, IE is a rare occurrence with decreasing mortality [6]. Accordingly, the indication of IE prevention for treatment of PDA was reviewed, as closure of small, hemodynamically non-significant PDA might not be necessary. Consequently, we hypothesized that the number of PDA closures might have decreased within the last decade, especially for small PDA.

The aim of this observational data analysis was to review the types of PDA closed, the devices used, success rates, immediate or postprocedural adverse events (AE) and the overall changing pattern of the procedure by comparing two time periods before and after changes in AP guidelines in 2009.

\section{Material and Methods}

\subsection{Patients}

In this single center descriptive observational study, procedure, outcome and follow-up of transcatheter PDA closure at the University Children's Hospital Zurich within the last 18 years were analyzed. Patients between January 2002 and September 2019 were included after agreement of their guardians, including patients with minor accompanying congenital heart disease (CHD). Patients without consent or with major associated CHD were excluded. Indication for PDA closure was given in PDA with hemodynamically relevant left-to-right shunt with clinical signs of pulmonary or cardiac congestion. In the past, reducing the risk of IE was another indication for closure even in asymptomatic patients. Demographic data, medical history, echocardiographic data and catheter intervention reports were taken from hospital databases. Follow-up results were obtained from our center as well as from referring outpatient pediatric cardiologists.

\subsection{PDA Morphology and Hemodynamics}

PDA morphology was determined by angiography and categorized as type A to E, according to the classification of Krichenko et al. [7]. Hemodynamic relevance was determined by Fick principle, relevant left-to-right shunt was defined by a Qp:Qs ratio of more than 1.5:1, which characterized hemodynamic indication for PDA closure.

\subsection{Procedure}

At our institution, transcatheter PDA closure was performed under general anesthesia with endotracheal intubation. Periinterventional AP was given, starting with a single dose of cefazolin ( $25 \mathrm{mg} / \mathrm{kg}$ body weight) which was repeated twice within $24 \mathrm{~h}$. After puncture of femoral artery and (if needed) femoral vein using ultrasound guidance, an introducer sheath was inserted and an intravenous bolus of unfractionated heparin (100 units $/ \mathrm{kg}$ ) was administered. Diagnostic catheters were used to assess hemodynamics by pressure recording and oxygen saturation measurements for shunt calculation, and vascular resistance calculations according to the Fick principle. Biplane angiography in the descending aorta (LAO $90^{\circ}$ and RAO 20 ${ }^{\circ}$ ) was performed to determine PDA morphology. Diameter at aortic and pulmonary end, minimal diameter and length were measured. A device was selected according to PDA morphology and size and delivered either from an antegrade (venous) or retrograde (arterial) approach. Last angiography confirmed final device position and documented degree of closure. After successful closure, AP was recommended for the next 6 months. 


\subsection{Follow-Up}

Results of transthoracic 2D-Doppler echocardiography performed on the day after intervention, 6 months later and at last available follow-up were evaluated. Trivial residual shunt was defined as color Doppler flow signal $<1 \mathrm{~mm}$ in the main pulmonary artery without hemodynamic significance, small residual shunt as signal $>1 \mathrm{~mm}$ with potential hemodynamic significance.

\subsection{Adverse Events}

AE were categorized according to a nomenclature proposed by Bergersen et al. [8] containing severity levels 1-5. AE level 3-5 was defined as relevant AE.

Procedure-related $A E$ included device embolization and malposition. Embolization was defined as a completely new position of the device outside the PDA after release, i.e., in the pulmonary artery or aorta. Device malposition was defined as a suboptimal final device position due to missizing without complete embolization. Definition of missizing was based on angiography, no balloon sizing was performed. We subdivided malposition as missizing due to mismatch between device and PDA size: either "too small" (leading to hemodynamically relevant residual shunt) or "too large" (leading to protrusion into the descending aorta or left pulmonary artery (LPA) and subsequent stenosis). Stenosis was defined as newly acquired turbulent Doppler flow pattern after PDA closure, determined by echocardiography with systolic Doppler flow velocity of $\geq 2 \mathrm{~m} / \mathrm{s}$.

Anesthesia-related $A E$ included medication error, airway problems or need for reintubation.

Vascular access-related $A E$ were defined as issues related to femoral artery or vein puncture such as thrombosis, aneurysm or prolonged bleeding.

Other $A E$ included those not mentioned above.

\subsection{Infective Endocarditis Antibiotic Prophylaxis for PDA}

We established a comparison of transcatheter PDA closure between two time periods: before (20022009) and after (2010-2019) changes in AP guidelines in Switzerland [5].

\subsection{Statistical Analysis}

Statistical analysis was performed using SPSS Statistics Version 25 (IBM Corp., Armonk, NY). Continuous variables (non-normally distributed) are described as median with interquartile range and analyzed using the Mann-Whitney $U$ Test. Categorical variables are described by frequencies and percentages and compared by means of the Chi-Square/Fisher's Exact Test. $p$-values below 0.05 were considered significant.

\subsection{Ethics}

Ethical approval was given by the cantonal ethics committee by protocol KEK-ZH-No. 2019-00583.

\section{Results}

\subsection{Patient Characteristics}

A total of 352 children and adolescents were treated by transcatheter PDA closure at the University Children's Hospital Zurich between January 2002 and September 2019. Consent for study participation was not available for 20 patients. Therefore, 332 patients were included in the analysis, whereof 226 (68.1\%) were female. Median age at cardiac catheter intervention was 3.0 years (interquartile range: $1.5-$ $5.7)$, with 53 patients (16.0\%) younger than one year. Body weight was $14.0 \mathrm{~kg}$ (10.0-19.3). Sixty-nine children were born preterm (20.8\%). Genetic syndromes were present in 43 patients $(13.0 \%)$, the most prevalent was Down syndrome in 21 patients $(6.3 \%)$. 


\subsection{Cardiac Comorbidity}

Cardiac comorbidity is shown in Table 1. Procedures combined with PDA closure were performed in 21 patients $(6.3 \%)$. These included procedures for secundum type atrial septal defect $(\mathrm{n}=10)$, aortic valve stenosis $(\mathrm{n}=2)$, pulmonary valve stenosis $(\mathrm{n}=1)$, coarctation of the aortic arch $(\mathrm{n}=2)$, native LPA stenosis $(\mathrm{n}=1$, shown in Fig. 1$)$, pulmonary sequestration artery $(\mathrm{n}=1)$ and major aortopulmonary collateral artery $(n=1)$. In three patients, myocardial biopsy was performed, confirming dilated cardiomyopathy in two cases.

Table 1: Cardiac comorbidities and combined procedures in patients undergoing transcatheter PDA closure

\begin{tabular}{|c|c|c|c|}
\hline Cardiac comorbidities & $\mathrm{n}(\%)$ & Combined procedures & $\mathrm{n}(\%)$ \\
\hline \multicolumn{4}{|l|}{ Left-to-right shunt CHD } \\
\hline Patent foramen ovale & $59(17.8)$ & & \\
\hline Secundum type atrial septal defect & $10(3)$ & ASD closure & $10(3)^{*}$ \\
\hline Ventricular septal defect, small & $10(3)$ & & \\
\hline \multicolumn{4}{|l|}{ Cardiac valve CHD } \\
\hline Bicuspid aortic valve & $13(3.9)$ & & \\
\hline Aortic valve stenosis & $7(2.1)$ & Aortic valve balloon valvuloplasty & $2(0.6)$ \\
\hline Aortic valve regurgitation & $6(1.8)$ & & \\
\hline Pulmonary valve stenosis & $2(0.6)$ & Pulmonary valve balloon valvuloplasty & $1(0.3)$ \\
\hline Mitral valve stenosis & $2(0.6)$ & & \\
\hline Mitral valve regurgitation & $1(0.3)$ & & \\
\hline Tricuspid valve regurgitation & $2(0.6)$ & & \\
\hline \multicolumn{4}{|l|}{ Vascular stenotic CHD } \\
\hline Native left pulmonary artery stenosis & $3(0.9)$ & LPA stenting & $1(0.3)$ \\
\hline \multirow[t]{2}{*}{ Coarctation of the aortic arch } & $2(0.6)$ & CoA angioplasty & $1(0.3)$ \\
\hline & & CoA stenting & $1(0.3)$ \\
\hline \multicolumn{4}{|l|}{ Other CHD } \\
\hline MAPCA & $7(2.1)$ & MAPCA coiling & $1(0.3)$ \\
\hline LSVC into coronary sinus & $5(1.5)$ & & \\
\hline Aberrant subclavian artery & $5(1.5)$ & & \\
\hline Dilated cardiomyopathy & $2(0.6)$ & Myocardial biopsy for suspected DCM** & $3(0.9)$ \\
\hline Total atrioventricular block & $1(0.3)$ & & \\
\hline WPW syndrome & $1(0.3)$ & & \\
\hline Pulmonary sequestration artery & $1(0.3)$ & Pulmonary sequestration artery coiling & $1(0.3)$ \\
\hline
\end{tabular}

\subsection{PDA Morphology and Hemodynamics}

The majority of PDA were categorized as conical type A $(n=167,50.3 \%)$ or tubular type $\mathrm{C}(\mathrm{n}=133$, $40.1 \%)$, followed by complex type $\mathrm{D}(\mathrm{n}=7,2.1 \%)$, window type $\mathrm{B}(\mathrm{n}=4,1.2 \%)$ and elongated type $\mathrm{E}(\mathrm{n}=3$, 
$0.9 \%)$. The remainder were residual shunts $(\mathrm{n}=5,1.5 \%$, three after surgical ligation, two after preceding intervention) or unclassified types of PDA $(n=13,3.9 \%)$. In six cases $(1.8 \%)$, a PDA aneurysm was documented. Median minimal PDA diameter was $1.9 \mathrm{~mm}(1.3-2.5)$, usually on the pulmonary artery side. Age and minimal diameter were inversely correlated $(r=-0.179, p=0.005)$. Aortic diameter of PDA was $6.3 \mathrm{~mm}$ (4.6-7.5) and PDA length was $8.0 \mathrm{~mm}$ (6.2-10.2). Median left-to-right shunt (Qp:Qs) was 1.67:1 (data available in $\mathrm{n}=183$ ). Mean pulmonary artery pressure (mPAP) was $20.0 \mathrm{mmHg}(18.0$ 23.0). A total of $53(18.5 \%)$ patients (data available in $\mathrm{n}=286$ ) met the criteria for pulmonary hypertension defined as mPAP $>25 \mathrm{mmHg}$. Larger PDA determined by minimal diameter was associated with higher rate of pulmonary hypertension with elevated $\operatorname{mPAP}(r=0.545, p<0.001)$.

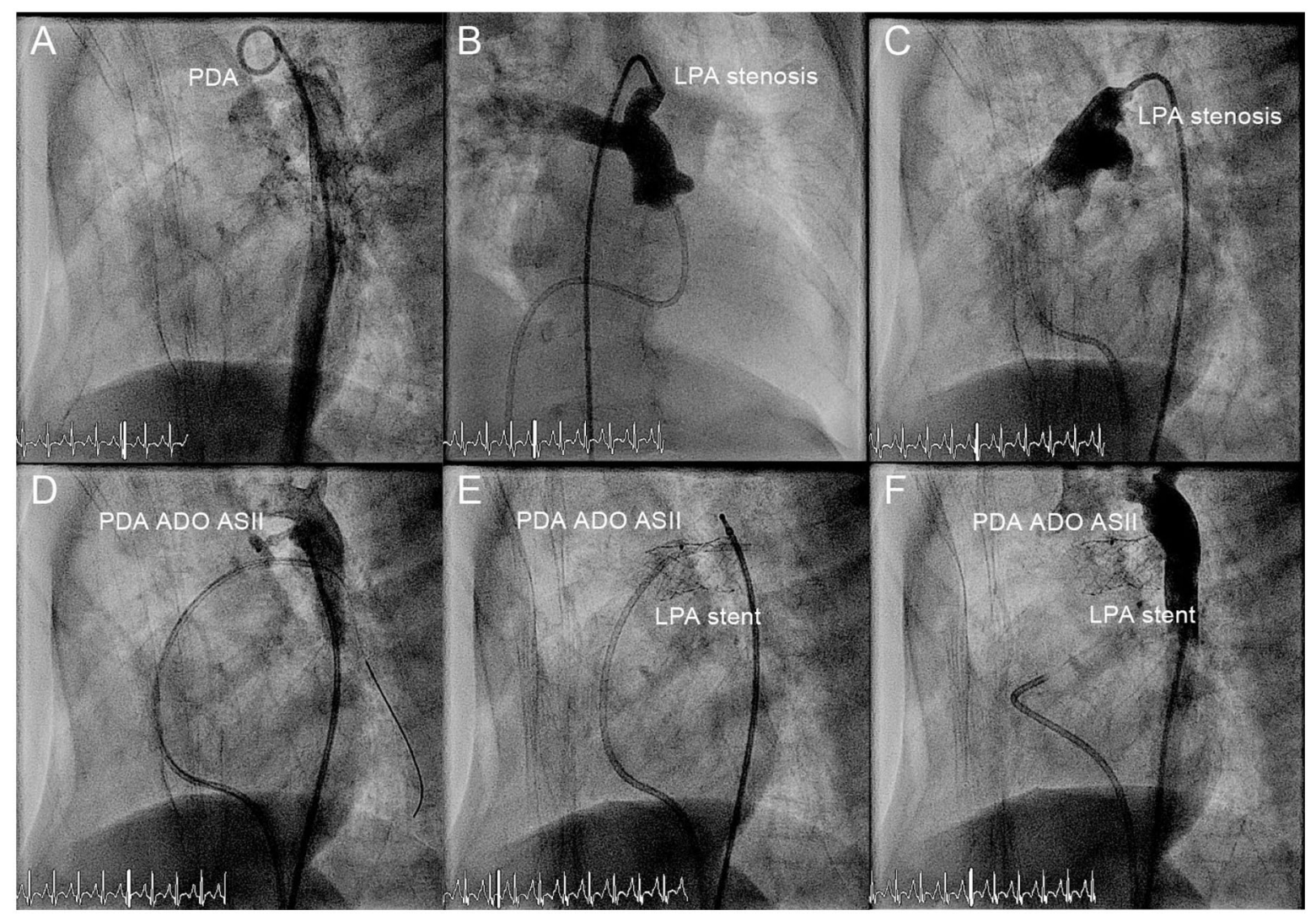

Figure 1: Combined transcatheter PDA closure (ADO II AS) and LPA stent placement (Palmaz Genesis). (A) Lateral view, PDA minimal size $2.5 \mathrm{~mm}$. (B) LAO $30^{\circ}$ cranial $20^{\circ}$, LPA stenosis. (C) Lateral view, LPA stenosis. (D) Lateral view, ADO II AS 5/6, positioned from descending aorta (retrograde), not released. (E) Lateral view, LPA stent placement Palmaz Genesis $10 \times 19 \mathrm{~mm}$. (F) Lateral view, ADO II AS released. Abbreviations: $A D O$ Amplatzer Duct Occluder, $A S$ additional sizes, $L P A$ left pulmonary artery, $P D A$ patent ductus arteriosus

\subsection{PDA Closure and Occluder Devices}

Median procedure time was 43.5 minutes (32.0-60.0), with a fluoroscopy time of 6.4 minutes (4.3$10.9)$, area dose product of $1.4 \mathrm{~Gy} \cdot \mathrm{cm}^{2}(0.6-2.5)$ and contrast medium quantity of $39.0 \mathrm{ml}(30.0-58.0)$. Used PDA devices and their sizes are shown in Table 2. Different types of Amplatzer Duct Occluders (ADO, Abbott Laboratories, Abbott Park, Illinois, USA) were used in 139 patients (41.9\%), including ADO I $(\mathrm{n}=124,37.3 \%)$, ADO II $(\mathrm{n}=9,2.7 \%)$ and ADO II additional sizes (AS) $(\mathrm{n}=6,1.8 \%)$. Coils 
were used in 187 patients (56.3\%), such as Cook (Cook Group Incorporated, Bloomington, Indiana, USA) detachable coils $(\mathrm{n}=177,53.3 \%$ ), Boston Scientific (Boston Scientific Corporation, Marlborough, Massachusetts, USA) pushable coils $(\mathrm{n}=6,1.8 \%)$ and Nit-Occlud (pfm medical AG, Cologne, Germany) PDA coils $(\mathrm{n}=4,1.2 \%)$. As other devices in 6 patients $(1.8 \%)$ we used Amplatzer Vascular Plug II $(\mathrm{n}=4,1.2 \%)$, Amplatzer Muscular Ventricular Septal Defect (VSD) Occluder $(\mathrm{n}=1,0.3 \%)$ and NitOcclud PDA-R $(\mathrm{n}=1,0.3 \%)$. More than one coil was used in 8 patients: two Cook detachable coils $(\mathrm{n}=6)$, three Cook detachable coils $(\mathrm{n}=1)$, and a Cook detachable coil combined with a Nit-Occlud PDA coil $(n=1)$. Not counted were patients with PDA closure using Boston Scientific pushable coils, where multiple use is routine.

Table 2: Devices used for transcatheter PDA closure in pediatric patients

\begin{tabular}{|c|c|c|c|c|}
\hline & Type of device & $\mathrm{n}(\%)$ & Size & $\mathrm{n}$ \\
\hline \multirow[t]{10}{*}{ ADO devices } & AmplatzerTM Duct Occluder & $124(37.3)$ & $5 / 4$ & 4 \\
\hline & & & $6 / 4$ & 74 \\
\hline & & & $8 / 6$ & 39 \\
\hline & & & $10 / 8$ & 7 \\
\hline & Amplatzer ${ }^{\mathrm{TM}}$ Duct Occluder II & $9(2.7)$ & $3 / 4$ & 7 \\
\hline & & & $4 / 4$ & 1 \\
\hline & & & $4 / 6$ & 1 \\
\hline & AmplatzerTM Duct Occluder II AS & $6(1.8)$ & $4 / 6$ & 1 \\
\hline & & & $5 / 4$ & 1 \\
\hline & & & $5 / 6$ & 4 \\
\hline \multirow[t]{14}{*}{ Coils } & Cook $^{\circledR}$ detachable coil & $177(53.6) *$ & $3 / 3$ & 43 \\
\hline & & & $3 / 4$ & 27 \\
\hline & & & $4 / 4$ & 1 \\
\hline & & & $3 / 5$ & 9 \\
\hline & & & $5 / 3$ & 10 \\
\hline & & & $5 / 4$ & 47 \\
\hline & & & $5 / 5$ & 44 \\
\hline & & & $5 / 6$ & 1 \\
\hline & & & $6.5 / 3$ & 1 \\
\hline & & & $6.5 / 5$ & 3 \\
\hline & Boston Scientific ${ }^{\circledR}$ pushable coil(s) & $6(1.8)$ & - & 6 \\
\hline & pfm Nit-Occlud ${ }^{\circledR}$ PDA coil & $4(1.2) * *$ & $5 \times 4$ & 1 \\
\hline & & & $6 \times 5$ & 2 \\
\hline & & & $7 \times 6$ & 1 \\
\hline \multirow[t]{4}{*}{ Others } & Amplatzer ${ }^{\mathrm{TM}}$ Vascular Plug II & $4(1.2)$ & $8 / 7$ & 3 \\
\hline & & & $10 / 7$ & 1 \\
\hline & Amplatzer ${ }^{\mathrm{TM}}$ Muscular VSD Occluder & $1(0.3)$ & 12 & 1 \\
\hline & $p f m$ Nit-Occlud ${ }^{\circledR}$ PDA-R & $1(0.3)$ & $7 / 12$ & 1 \\
\hline
\end{tabular}

Note: *In 7 patients, more than one Cook detachable coil was implanted.

**In one patient, a Cook detachable coil was implanted in addition to Nit-Occlud PDA coil.

Abbreviations: $A D O$ Amplatzer Duct Occluder, $A S$ additional sizes, $n$ number, $P D A$ patent ductus arteriosus, $V S D$ ventricular septal defect. 
A comparison of the three device groups is shown in Table 3. Compared to coils, ADO devices were more frequently used in younger and smaller children $(1.8 v s .3 .9$ years, $11.0 v s .16 .4 \mathrm{~kg} ; p<0.001$ for both), more often in type A and B PDA morphology $(p<0.001)$, for larger PDA (minimal diameter 2.4 vs. $1.2 \mathrm{~mm}, p<0.001)$ and higher mPAP $(21.0 v s .19 .0 \mathrm{mmHg}, p<0.001)$, needed longer procedure time (47.5 vs. 35.0 minutes, $p<0.001)$ and had a high number of complete closure rate at last follow-up $(100 \%$ vs. $97.1 \%, p=0.040)$. Of note, age and body weight were particularly low in patients receiving ADO II or ADO II AS (1.1 years and $8.4 \mathrm{~kg}$ ). In patients treated with more than one coil, median procedure time was 72.0 minutes and fluoroscopy time 17.1 minutes. Devices beside ADO and coils grouped as "others" were implanted in significantly larger $(4.3 \mathrm{~mm} v s .1 .9 \mathrm{~mm}, p<0.001)$ and longer PDA (16.8 mm vs. $8.0 \mathrm{~mm}, p=0.029)$ with higher mPAP $(27.0 \mathrm{mmHg} v s .20 .0 \mathrm{mmHg}, p=0.001)$ and needed longer procedure time (87.0 minutes vs. 43.0 minutes, $p=0.018$ ). Relevant AE (level 3-5) did not differ significantly between the groups.

Table 3: Comparison of different device types for transcatheter PDA closure in pediatric patients

\begin{tabular}{|c|c|c|c|c|c|c|}
\hline Variable & Total & ADO Devices & Coils & Others & $p$-value & $p$-value \\
\hline Devices & & $\begin{array}{l}\text { ADO I } \\
\text { ADO II } \\
\text { ADO II AS }\end{array}$ & $\begin{array}{l}\text { Cook detachable coil } \\
\text { BS pushable coil } \\
\text { Nit-Occlud PDA coil }\end{array}$ & $\begin{array}{l}\text { AVP II } \\
\text { VSD Occluder } \\
\text { Nit-Occlud PDA-R }\end{array}$ & $\begin{array}{l}\text { ADO } \\
v s . \\
\text { Coils }\end{array}$ & $\begin{array}{l}\text { Others } \\
v s . \\
\text { ADO + } \\
\text { Coils }\end{array}$ \\
\hline Patients & 332 & $139(41.9)$ & $187(56.3)$ & $6(1.8)$ & & \\
\hline Age (y) & $3.0(1.5-5.7)$ & $1.8(0.9-3.7)$ & $3.9(2.4-6.5)$ & $2.4(1.3-3.3)$ & $<0.001$ & 0.572 \\
\hline Weight (kg) & $14.0(10.0-19.3)$ & $11.0(8.6-16.0)$ & $16.4(12.1-23.0)$ & $12.0(10.9-14.0)$ & $<0.001$ & 0.562 \\
\hline Type of PDA & & & & & $<0.001$ & 0.728 \\
\hline Type A (conical) & $167(50.3)$ & $96(69.1)$ & $69(36.9)$ & $2(33.3)$ & & \\
\hline Type B (window) & $4(1.2)$ & $4(2.9)$ & $0(0.0)$ & $0(0.0)$ & & \\
\hline Type C (tubular) & $133(40.1)$ & $38(27.3)$ & $92(49.2)$ & $3(50.0)$ & & \\
\hline Type D (complex) & $7(2.1)$ & $1(0.7)$ & $6(3.2)$ & $0(0.0)$ & & \\
\hline Type E (elongated) & $3(0.9)$ & $0(0.0)$ & $3(1.6)$ & $0(0.0)$ & & \\
\hline Unknown/Residual & $18(5.4)$ & $0(0.0)$ & $17(9.1)$ & $1(16.7)$ & & \\
\hline Minimal PDA diameter & $1.9(1.3-2.5)$ & $2.4(2.0-2.8)$ & $1.2(1.0-1.5)$ & $4.3(3.1-4.9)$ & $<0.001$ & $<0.001$ \\
\hline PDA length & $8.0(6.2-10.2)$ & $7.8(6.2-10.0)$ & $8.0(6.2-10.8)$ & $16.8(14.6-18.9)$ & 0.520 & 0.029 \\
\hline mPAP $(\mathrm{mmHg})$ & $20.0(18.0-23.0)$ & $21.0(19.0-25.0)$ & $19.0(17.0-22.0)$ & $27.0(25.3-42.3)$ & $<0.001$ & 0.001 \\
\hline Procedure time (min) & $43.5(32.0-60.0)$ & $47.5(39.0-60.0)$ & $35.0(28.0-58.0)$ & $87.0(49.0-96.0)$ & $<0.001$ & 0.018 \\
\hline Fluoroscopy time (min) & $6.4(4.3-10.9)$ & $6.5(5.1-9.4)$ & $5.8(3.6-11.2)$ & $12.6(6.7-18.4)$ & 0.122 & 0.059 \\
\hline $\operatorname{ADP}\left(\mathrm{Gy} \cdot \mathrm{cm}^{2}\right)$ & $1.4(0.6-2.5)$ & $1.1(0.6-2.1)$ & $1.4(0.7-3.0)$ & $2.4(1.0-3.0)$ & 0.038 & 0.300 \\
\hline Contrast medium (ml) & $39.0(30.0-58.0)$ & $39.5(32.0-60.0)$ & $36.0(26.0-50.0)$ & $40.0(33.8-58.3)$ & 0.024 & 0.619 \\
\hline Relevant AE (level 3-5) & $15(4.5)$ & $7(5.0)$ & $8(4.3)$ & $0(0.0)$ & 0.747 & 1.000 \\
\hline
\end{tabular}

Note: Data are given as number (\%) or median (interquartile range).

Abbreviations: $A D O$ Amplatzer duct occluder, $A D P$ area dose product, $A E$ adverse events, $B S$ Boston Scientific, $F U$ follow-up, $m P A P$ mean pulmonary artery pressure, $P D A$ patent ductus arteriosus, VSD Occluder Amplatzer Muscular Ventricular Septal Defect Occluder, Qp:Qs Ratio of pulmonary blood flow to systemic blood flow.

\subsection{PDA Closure Rates}

The postprocedural course and closure rate are shown in Fig. 2. In three patients, the intervention was aborted and switched to surgery $(n=2)$ or postponed to later device closure $(n=1)$. Of all patients with implanted devices $(n=329)$, complete PDA closure was observed by angiography at the end of catheter intervention in 201 (61.1\%). On echocardiography the day after, PDA was completely closed in 
240 patients $(72.6 \%)$, trivial residual shunt was found in $60(18.2 \%)$, small in $23(7.0 \%)$, and PDA recanalization due to embolization in $4(1.2 \%)$ (no data available $\mathrm{n}=2$ ). Further follow-up was available in 308 out of 329 patients $(93.6 \%)$, with last follow-up at a median of 3.3 years (1.0-6.9). Complete closure was shown in 270 of $308(87.7 \%)$ by follow-up at 6 months (trivial shunt $\mathrm{n}=27$, small shunt $\mathrm{n}=9$, referral to surgery $\mathrm{n}=1$, no data available, $\mathrm{n}=1$ ). In three patients, secondary catheter intervention was performed for small hemodynamically relevant residual shunts $(1.2,1.5$ and 5.7 years after first intervention). Another secondary intervention was performed for late embolization after 4.5 years. At last follow-up, closure had occurred in 302 out of 307 patients $(98.4 \%$ ) (trivial shunt $\mathrm{n}=4$, small shunt $\mathrm{n}=1$ ). All 5 patients with residual shunts were treated with Cook detachable coils.

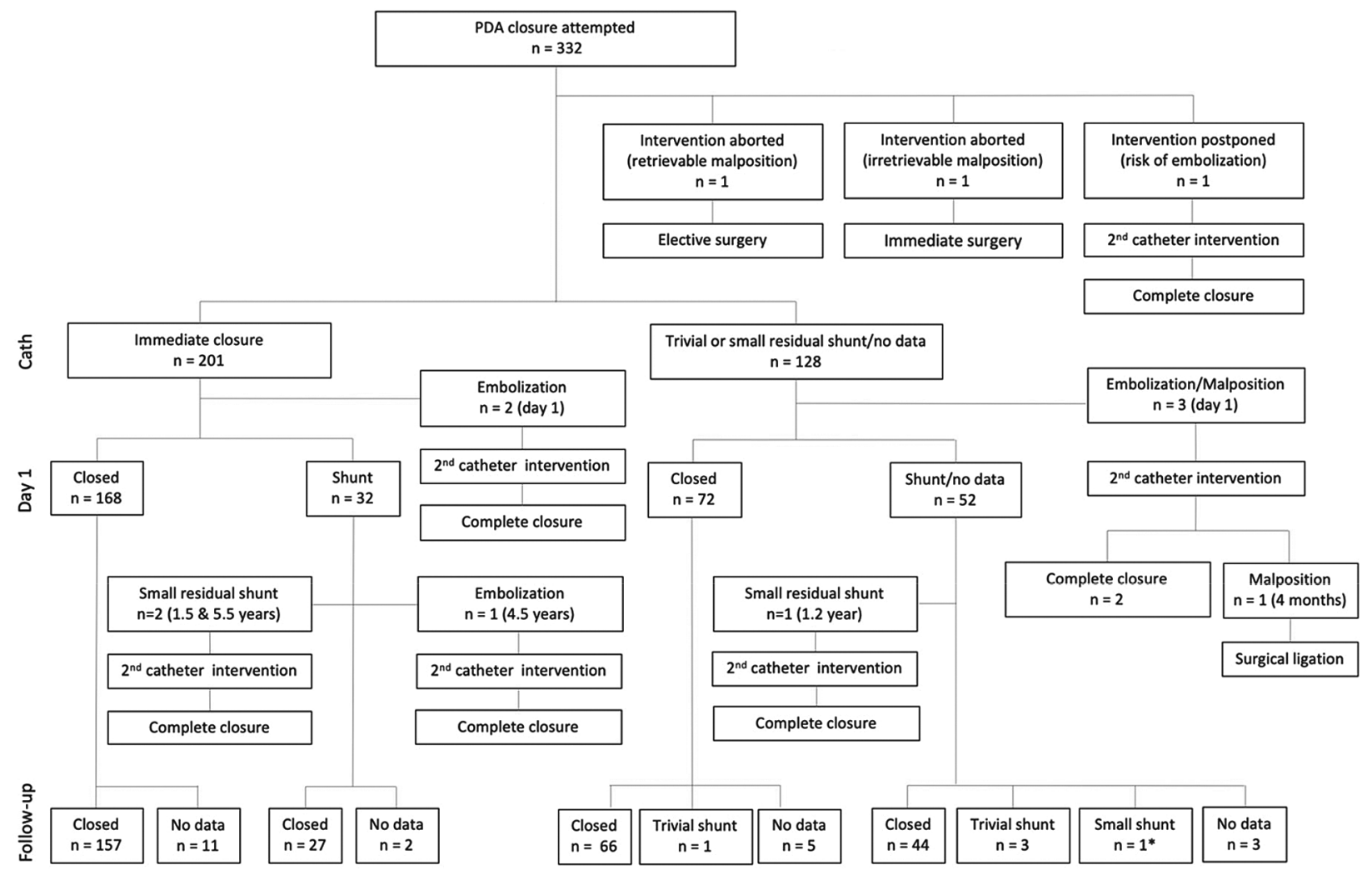

Figure 2: Closure rates and postprocedural course after transcatheter PDA closure in children.

* Secondary intervention recommended.

Abbreviations: cath catheter intervention, $P D A$ patent ductus arteriosus

\subsection{Adverse Events}

Overall, we found AE in 57 of 332 patients (17.2\%), with severity level 1 (mild) in 17 patients (5.12\%), level 2 (minor) in 25 patients (7.5\%), level 3 (moderate) in 14 patients (4.2\%), level 4 (major) in 1 patient $(0.3 \%)$ and level 5 (catastrophic) in 0 patients $(0 \%)$. Patients under one year of age were at higher risk for AE (21/53 patients, $39.6 \%$, vs. $35 / 279$ patients, $12.5 \%$; $p<0.001$ ). Relevant AE defined as severity level $3-$ 5 occurred in $4.5 \%$ of patients, more often in infants as well $(9.4 \% v s .2 .9 \%, p=0.024)$.

Procedure-related $A E$. Procedure-related AE requiring corrective measures due to device malposition or embolization are listed in Table 4. Device missizing "too small" registered during the intervention $(\mathrm{n}=14)$ was mostly solved by retrieval and change $(n=5)$ or use of a secondary device ("device in device") $(n=8)$. In one patient, a device of appropriate size was not available, and a secondary closure was performed one month 
later. Device missizing "too small" after the intervention $(\mathrm{n}=4)$ led to hemodynamically relevant residual shunt closed by insertion of a second coil $(n=3$, lost to follow-up $n=1)$. Device missizing "too large" registered during the intervention $(n=5)$ was solved by retrieval and change $(n=2)$, optimization of device position by LPA balloon dilatation $(\mathrm{n}=1)$, immediate retrieval and elective surgical PDA closure $(n=1)$ or emergency surgical retrieval and PDA closure $(n=1)$. In one patient, missizing "too large" led to postprocedural aortic obstruction and was treated with secondary transcatheter intervention, device retrieval and closure using a smaller device. Missizing "too large" not necessitating further intervention but leading to descending aortic arch obstruction $(n=12)$ or LPA obstruction $(n=5)$ occurred with ADO devices and Amplatzer Muscular VSD Occluder, mainly in small children (median age 1.1 years, weight $7.8 \mathrm{~kg}$ ). In 10 of them, increased flow velocity was no longer documented at last follow-up, whereas peak flow velocity was $V_{\max } 2-3 \mathrm{~m} / \mathrm{s}$ in 6 and $V_{\max }>3 \mathrm{~m} / \mathrm{s}$ in one. In case of intraprocedural embolization $(\mathrm{n}=3)$, devices were retrieved and another device was successfully implanted. Postprocedural device embolization $(n=5)$ led to secondary intervention with device retrieval and successful complete closure.

Table 4: Adverse events during and after transcatheter PDA closure due to device malposition or embolization requiring corrective measures

\begin{tabular}{|c|c|c|c|c|c|c|c|}
\hline & Problem & $1^{\text {st }}$ device & Solution & $\begin{array}{l}\text { New } \\
\text { approach }\end{array}$ & Time & PDA type & Severity \\
\hline \multicolumn{8}{|c|}{ During intervention } \\
\hline & \multicolumn{7}{|c|}{ Malposition "too large" (Device > PDA) } \\
\hline$\# 1$ & $\begin{array}{l}\text { Malposition leading to LPA } \\
\text { stenosis }\end{array}$ & ADO I $10 / 8$ & $\begin{array}{l}\text { Immediate surgery with device retrieval } \\
\text { and PDA closure }\end{array}$ & Surgery & immediate & D - complex & level 4 \\
\hline$\# 2$ & $\begin{array}{l}\text { Malposition leading to LPA } \\
\text { stenosis }\end{array}$ & $\begin{array}{l}\text { Nit-Occlud } \\
\text { coil } 5 / 4\end{array}$ & $\begin{array}{l}\text { Location of device improved by LPA } \\
\text { balloon dilatation }\end{array}$ & - & immediate & A - conical & level 2 \\
\hline \#3 & $\begin{array}{l}\text { Malposition leading to } \\
\text { aortic arch stenosis }\end{array}$ & ADO I $8 / 6$ & Elective surgery with PDA closure & Surgery & 1 month & B - window & level 3 \\
\hline$\# 4$ & $\begin{array}{l}\text { Malposition leading to } \\
\text { aortic arch stenosis }\end{array}$ & Cook coil 5/4 & Interventional retrieval and PDA closure & $\begin{array}{l}\text { Cook coil } \\
3 / 4\end{array}$ & immediate & $\mathrm{C}$ - tubular & level 2 \\
\hline \multirow[t]{2}{*}{ \# 5} & $\begin{array}{l}\text { Malposition leading to } \\
\text { aortic arch stenosis }\end{array}$ & ADO II $4 / 4$ & Interventional retrieval and PDA closure & ADO I $6 / 4$ & immediate & A - conical & level 2 \\
\hline & \multicolumn{7}{|c|}{ Malposition "too small" (Device $<$ PDA) } \\
\hline$\# 6$ & $\begin{array}{l}\text { Unstable, suitable device } \\
\text { not available }\end{array}$ & ADO I $8 / 6$ & $\begin{array}{l}\text { Interventional retrieval, secondary } \\
\text { intervention and PDA closure }\end{array}$ & ADO II $6 / 6$ & 1 month & $\mathrm{C}$ - tubular & level 3 \\
\hline \# 7 & Unstable & ADO I $6 / 4$ & Interventional retrieval and PDA closure & ADO I $8 / 6$ & immediate & $\mathrm{B}$ - window & level 2 \\
\hline$\# 8$ & Unstable & ADO I 12/10 & Interventional retrieval and PDA closure & $\begin{array}{l}\text { VSD Occ. } \\
12\end{array}$ & immediate & A - conical & level 2 \\
\hline$\# 9$ & Unstable & ADO I $6 / 4$ & Interventional retrieval and PDA closure & $\begin{array}{l}\text { Cook coil } \\
5 / 5\end{array}$ & immediate & $\mathrm{C}$ - tubular & level 2 \\
\hline \# 10 & Residual shunt & ADO I $8 / 6$ & Interventional retrieval and PDA closure & $\begin{array}{l}\text { ADO I } 10 / \\
8\end{array}$ & immediate & A - conical & level 2 \\
\hline \# 11 & Residual shunt & Cook coil 5/5 & Interventional retrieval and PDA closure & ADO I 6/4 & immediate & A - conical & level 2 \\
\hline \# 12 & Residual shunt & $\begin{array}{l}\text { Cook coil } \\
6.5 / 5\end{array}$ & $\begin{array}{l}\text { Interventional PDA closure by additional } \\
\text { coil implantation }\end{array}$ & $\begin{array}{l}\text { Cook coil } \\
3 / 4\end{array}$ & immediate & A - conical & level 2 \\
\hline \# 13 & Residual shunt & $\begin{array}{l}\text { Nit-Occlud } \\
\text { coil } 6 / 7\end{array}$ & $\begin{array}{l}\text { Interventional PDA closure by additional } \\
\text { coil implantation }\end{array}$ & $\begin{array}{l}\text { Cook coil } \\
5 / 4\end{array}$ & immediate & A - conical & level 2 \\
\hline \# 14 & Residual shunt & Cook coil 5/5 & $\begin{array}{l}\text { Interventional PDA closure by additional } \\
\text { coil implantation }\end{array}$ & $\begin{array}{l}\text { Cook coil } \\
5 / 4\end{array}$ & immediate & A - conical & level 2 \\
\hline \# 15 & Residual shunt & Cook coil $5 / 5$ & $\begin{array}{l}\text { Interventional PDA closure by additional } \\
\text { coil implantation }\end{array}$ & $\begin{array}{l}\text { Cook coil } \\
5 / 4\end{array}$ & immediate & A - conical & level 2 \\
\hline \# 16 & Residual shunt & Cook coil $5 / 3$ & $\begin{array}{l}\text { Interventional PDA closure by additional } \\
\text { coil implantation }\end{array}$ & $\begin{array}{l}\text { Cook coil } \\
3 / 3\end{array}$ & immediate & $\mathrm{C}-$ tubular & level 2 \\
\hline
\end{tabular}




\begin{tabular}{|c|c|c|c|c|c|c|c|}
\hline & Problem & $1^{\text {st }}$ device & Solution & $\begin{array}{l}\text { New } \\
\text { approach }\end{array}$ & Time & PDA type & Severity \\
\hline \# 17 & Residual shunt & Cook coil 5/4 & $\begin{array}{l}\text { Interventional PDA closure by additional } \\
\text { coil implantation }\end{array}$ & $\begin{array}{l}\text { Cook coil } \\
5 / 3\end{array}$ & immediate & A - conical & level 2 \\
\hline \# 18 & Residual shunt & $\begin{array}{l}\text { Cook coil } \\
6.5 / 5\end{array}$ & $\begin{array}{l}\text { Interventional PDA closure by additional } \\
\text { coil implantation }\end{array}$ & $\begin{array}{l}\text { Cook coil } \\
6.5 / 3\end{array}$ & immediate & A - conical & level 2 \\
\hline \multirow[t]{2}{*}{ \# 19} & Residual shunt & Cook coil $5 / 5$ & $\begin{array}{l}\text { Interventional PDA closure by additional } \\
\text { coil implantation }\end{array}$ & $\begin{array}{l}2 \times \text { Cook } \\
\text { coil } 5 / 4\end{array}$ & immediate & $\mathrm{D}$ - complex & level 2 \\
\hline & Device embolization & & & & & & \\
\hline \# 20 & $\begin{array}{l}\text { Intraprocedural } \\
\text { embolization into LPA }\end{array}$ & $\begin{array}{l}\text { BS pushable } \\
\text { coil }\end{array}$ & $\begin{array}{l}\text { Interventional retrieval (one coil not } \\
\text { retrieved) and PDA closure }\end{array}$ & $\begin{array}{l}\text { Cook coil } \\
3 / 3\end{array}$ & immediate & D - complex & level 2 \\
\hline \# 21 & $\begin{array}{l}\text { Intraprocedural } \\
\text { embolization into LPA }\end{array}$ & $\begin{array}{l}\text { Cook coils } \\
5 / 3+5 / 4\end{array}$ & Interventional retrieval and PDA closure & ADO I $6 / 4$ & immediate & A - conical & level 2 \\
\hline \multirow[t]{3}{*}{ \# 22} & $\begin{array}{l}\text { Intraprocedural } \\
\text { embolization into LPA }\end{array}$ & $\begin{array}{l}\text { ADO II AS } \\
5 / 4\end{array}$ & Interventional retrieval and PDA closure & ADO I $8 / 6$ & immediate & A - conical & level 2 \\
\hline & & & After intervention & & & & \\
\hline & Malposition "too large & evice $>$ PDA) & & & & & \\
\hline \multirow[t]{2}{*}{ \# 23} & $\begin{array}{l}\text { Malposition leading to } \\
\text { aortic arch stenosis }\end{array}$ & ADO I $10 / 8$ & $\begin{array}{l}\text { Secondary cath. intervention with retrieval } \\
\text { and PDA closure }\end{array}$ & ADO I $8 / 6$ & 1 day & $\mathrm{C}$ - tubular & level 3 \\
\hline & Malposition "too small & evice $<$ PDA) & & & & & \\
\hline \# 24 & Residual shunt & Cook coil 5/3 & $\begin{array}{l}\text { Secondary cath. intervention and PDA } \\
\text { closure }\end{array}$ & $\begin{array}{l}\text { Cook coil } \\
3 / 3\end{array}$ & 1.5 years & $\mathrm{C}$ - tubular & level 3 \\
\hline \# 25 & Residual shunt & Cook coil 5/4 & $\begin{array}{l}\text { Secondary cath. intervention and PDA } \\
\text { closure }\end{array}$ & $\begin{array}{l}\text { Cook coil } \\
5 / 3\end{array}$ & 1.2 years & A - conical & level 3 \\
\hline \# 26 & Residual shunt & Cook coil 5/4 & $\begin{array}{l}\text { Secondary cath. intervention and PDA } \\
\text { closure }\end{array}$ & $\begin{array}{l}\text { Cook coil } \\
5 / 4\end{array}$ & 5.7 years & A - conical & level 3 \\
\hline \multirow[t]{2}{*}{ \# 27} & Residual shunt & Cook coil 5/4 & $\begin{array}{l}\text { Secondary cath. intervention advised, lost } \\
\text { to follow-up }\end{array}$ & $\begin{array}{l}\text { No data } \\
\text { available }\end{array}$ & 1.5 years & A - conical & level 3 \\
\hline & Device embolization & & & & & & \\
\hline \# 28 & $\begin{array}{l}\text { Postprocedural } \\
\text { embolization into LPA }\end{array}$ & Cook coil 3/4 & $\begin{array}{l}\text { Secondary cath. intervention, coil not } \\
\text { retrieved, PDA closure }\end{array}$ & $\begin{array}{l}\text { Cook coil } \\
5 / 4\end{array}$ & 1 day & $\mathrm{C}$ - tubular & level 3 \\
\hline \# 29 & $\begin{array}{l}\text { Postprocedural } \\
\text { embolization into LPA }\end{array}$ & Cook coil 3/4 & $\begin{array}{l}\text { Secondary cath. intervention, coil not } \\
\text { retrieved, PDA closure }\end{array}$ & $\begin{array}{l}\text { ADO II AS } \\
5 / 4\end{array}$ & 4.5 years & $\mathrm{C}$ - tubular & level 3 \\
\hline \# 30 & $\begin{array}{l}\text { Postprocedural } \\
\text { embolization into LPA }\end{array}$ & Cook coil 5/4 & $\begin{array}{l}\text { Secondary cath. intervention with retrieval } \\
\text { and PDA closure }\end{array}$ & ADO I $6 / 4$ & 1 day & $\mathrm{C}$ - tubular & level 3 \\
\hline \# 31 & $\begin{array}{l}\text { Postprocedural } \\
\text { embolization into LPA }\end{array}$ & ADO I $8 / 6$ & $\begin{array}{l}\text { Secondary cath. intervention with retrieval } \\
\text { and PDA closure }\end{array}$ & AVP II 8 & 1 day & $\mathrm{C}$ - tubular & level 3 \\
\hline \# 32 & $\begin{array}{l}\text { Postprocedural } \\
\text { embolization into LPA }\end{array}$ & $\begin{array}{l}\text { ADO II AS } \\
6 / 5\end{array}$ & $\begin{array}{l}\text { Secondary cath. intervention with retrieval } \\
\text { and PDA closure * }\end{array}$ & ADO I $8 / 6$ & 1 day & $\mathrm{C}$ - tubular & level 3 \\
\hline
\end{tabular}

Vascular access-related AE. These included transient thrombosis $(\mathrm{n}=5)$, pseudoaneurysm $(\mathrm{n}=1)$ and prolonged bleeding $(\mathrm{n}=1)$. They more frequently affected patients under 1 year of age $(4 / 53$ patients, $7.5 \%$ vs. $3 / 279,1.1 \% ; p=0.003)$.

Anesthesia-related AE. Pulmonary hypertensive crisis occurred in two patients, which was treated by intensifying mechanical ventilation and drug treatment. These two patients were small infants (7 and 8 months old, body weight $<6 \mathrm{~kg}$ ), one of them with Down syndrome. Pericardial effusion with spontaneous relief $(n=5)$ correlated mostly with volume overload during the intervention. 
Other AE. A very rare AE was recurrent laryngeal nerve paralysis $(\mathrm{n}=1)$. This was a preterm infant, in whom a tubular PDA was closed with Cook detachable coil at 5 months of age and body weight of $5.3 \mathrm{~kg}$. Within two weeks after discharge from hospital, it developed hoarseness, sucking weakness, difficulties in swallowing and failure to thrive, and laryngoscopy confirmed left-sided vocal cord dysfunction due to recurrent laryngeal nerve paralysis at the next consultation, one month after the intervention. After one year, there was complete symptom relief with normal vocal articulation and good body weight gain.

\subsection{Secondary Cardiac Catheterization and Surgical Procedure}

In total, secondary cardiac catheterization was performed or recommended in 11 patients (3.3\%) between one day and 5.7 years after first procedure. Reasons for secondary procedure were device embolization $(\mathrm{n}=5)$, missizing "too small" with residual shunt $(\mathrm{n}=4)$, or missizing "too large" with aortic obstruction $(n=2)$. Surgical procedure was needed in three patients $(0.9 \%)$, due to device malposition (missizing "too large") during the intervention $(\mathrm{n}=1)$, after one month $(\mathrm{n}=1)$ and four months after a secondary transcatheter intervention $(\mathrm{n}=1)$.

\subsection{The Effect of AP Guideline Change}

The comparison between the two periods before (2002-2009) and after (2010-2019) AP guideline change is shown in Table 5. The annual rate of PDA closures decreased (22.9 per year before vs. 15.5 after, $p=0.018$ ) (Fig. 3). In the second period, PDA were larger (2.0 vs. $1.6 \mathrm{~mm}, p=0.002)$, longer (8.9 vs. $7.0 \mathrm{~mm}, p=0.046)$, more often tubular type C (51.7\% vs. 33.7\%) than conical type A (47.0\% vs. $58.9 \%)(p=0.002)$ and more often closed with ADO devices $(59.6 \%$ vs. $27.1 \%)$ compared to coils $(37.1 \%$ vs. $72.4 \%)(p<0.001)$.

Table 5: Comparison of case characteristics before and after change in AP guidelines

\begin{tabular}{llll}
\hline Variable & Period 1 & Period 2 & $p$-value \\
\hline Time period & $01 / 2002-12 / 2009$ & $01 / 2010-09 / 2019$ & \\
Total number of interventions & 181 & 151 & 0.018 \\
Interventions per year & 22.9 & 15.5 & 0.510 \\
Female & $126(69.6)$ & $100(66.2)$ & 0.075 \\
Age (y) & $3.1(1.8-5.9)$ & $2.8(1.2-5.1)$ & 0.159 \\
Weight (kg) & $14.7(10.4-20.0)$ & $13.5(9.8-19.0)$ & 0.002 \\
Type of PDA (Krichenko) & & & \\
Type A (conical) & $96(53.0)$ & $71(47.0)$ & \\
Type B (window) & $3(1.7)$ & $1(0.7)$ & \\
Type C (tubular) & $55(30.4)$ & $78(51.7)$ & \\
Type D (complex) & $6(3.3)$ & $1(0.7)$ & \\
Type E (elongated) & $3(1.7)$ & $0(0.0)$ & 0.002 \\
$\quad$ Unknown/Residual & $18(9.9)$ & $0(0.0)$ & 0.046 \\
Minimal PDA diameter (mm) & $1.6(1.0-2.3)$ & $2.0(1.5-2.7)$ & 0.277 \\
PDA length (mm) & $7.0(5.9-8.6)$ & $8.9(6.4-11.0)$ & 0.835 \\
mPAP (mmHg) & $20.0(17.0-23.0)$ & $20.0(18.0-24.0)$ & $($ Continued) \\
Procedure time (min) & $45(30.0-63.0)$ & $43.0(34.0-57.5)$ & \\
\hline
\end{tabular}




\begin{tabular}{llll}
\hline Table 5 (continued). & & & \\
\hline Variable & Period 1 & Period 2 & $p$-value \\
\hline Fluoroscopy time (min) & $7.4(4.2-11.9)$ & $6.1(4.5-9.4)$ & 0.247 \\
Area dose product $\left(\mathrm{Gy} \cdot \mathrm{cm}^{2}\right)$ & $1.8(0.8-4.0)$ & $0.9(0.6-1.8)$ & $<0.001$ \\
Contrast medium (ml) & $36.0(27.0-51.0)$ & $40.0(32.0-60.0)$ & 0.040 \\
Device type & & & $<0.001$ \\
$\quad$ ADO device & $49(27.1)$ & $90(59.6)$ & \\
Coil & $131(72.4)$ & $56(37.1)$ & \\
$\quad$ Other & $1(0.6)$ & $5(3.3)$ & 0.925 \\
Relevant AE (level 3-5) & $8(4.4)$ & $7(4.6)$ & \\
\hline
\end{tabular}

Note: Data are given as number (\%) or median (interquartile range).

Abbreviations: $A D O$ Amplatzer Duct Occluder, $A E$ adverse events, $A P$ antibiotic prophylaxis, $F U$ follow-up, $m P A P$ mean pulmonary artery pressure, $P D A$ patent ductus arteriosus, $Q p: Q s$ ratio of pulmonary blood flow to systemic blood flow.

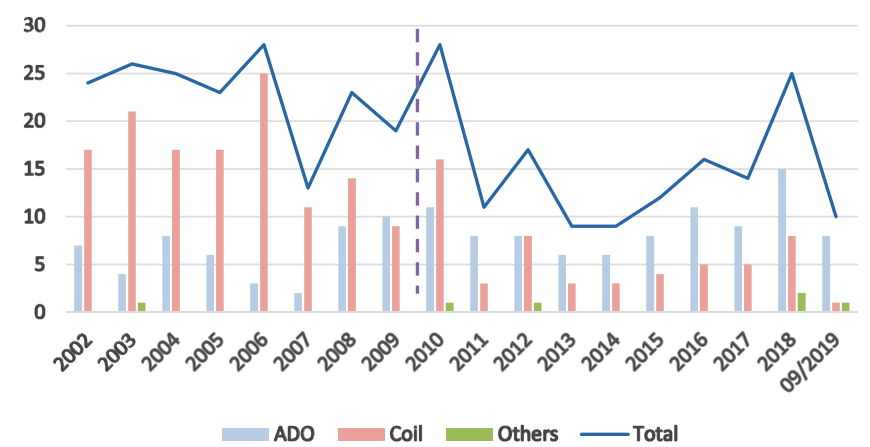

Figure 3: Annual rate of transcatheter PDA closures and different device types used over time. Abbreviations: $A D O$ Amplatzer Duct Occluder, $P D A$ patent ductus arteriosus

\section{Discussion}

Since first successful transcatheter PDA closure in 1967 [9], this minimally invasive technique has evolved into the standard of care [10]. The results in our cohort confirm that transcatheter PDA closure is a safe and efficient procedure with high rate of definite PDA closure at long-term follow-up (98.4\%). There was no procedure-related mortality, and a low number of moderate (4.2\%) and major (0.3\%) AE. According to changes in AP guidelines, the absolute number of PDA closures decreased in the last decade with a shift towards closure of larger PDA with need for larger devices.

Regarding the distribution of PDA morphologies, we predominantly found type A (conical) and C (tubular) in our cohort, type E (elongated) PDA was less frequent compared to other publications [11,12]. As discrimination between type $\mathrm{C}$ and $\mathrm{E}$ may be difficult, "borderline elongated" PDA might have been categorized as tubular. Type E often arises in the context of preterm infants [2]. However, introduction of a new fetal type $\mathrm{F}$ has been proposed, reserved for children born prematurely with long, wide and tortuous PDA [13]. In our sample, PDA morphologies of preterm and term children were similar, as type $\mathrm{F}$ has so far not been included and type $\mathrm{E}$ was rather uncommon.

Regarding devices used for PDA closure, several differences are highlighted. Compared to coils, ADO is rather used in younger children at lower body weight, for conical type A and window type B and for larger PDA, with longer procedure time but comparable radiation exposure. ADO devices include the cone-shaped first ADO I introduced in 1998, designed for closure of moderate-to-large-sized PDA [14]. In 2008, first 
results about the ADO II have been published, a device with two symmetrical retention discs and a thin waist allowing both ante- and retrograde delivery, especially for smaller children with small-to-moderate-sized PDA [15]. The later modification ADO II AS is used since 2012 with a central part only slightly less wide than the retention discs [16]. These newer devices have been developed for closure of a broader variety of PDA types, and the ADO II AS is produced in sizes suitable for premature newborns and small infants, attempting to avoid LPA or aortic arch obstruction frequently observed with ADO I [17]. This advantage of ADO II and ADO II AS is confirmed by our results. The ADO I has the well-known limitation of the large retention disk leading to aortic/LPA protrusion especially in small children with short PDA length [18]. Of note, in our cohort the majority of patients with this AE remained asymptomatic with no blood pressure gradient between upper and lower extremity and decreasing Doppler flow acceleration with further body growth, while two patients were referred to surgery after intervention and secondary intervention was performed in one.

Different types of coils, primarily Cook detachable coils, were used for closure of tubular type C, complex type D and elongated type E PDA. In our cohort, the main problem when using coils was residual PDA, with either trivial shunts not necessitating intervention $(n=4)$, or small but hemodynamically relevant shunts with need for secondary catheter intervention during mid-term followup $(\mathrm{n}=4)$, similar to other studies [19].

In some cases, with very large or long PDA morphologies, devices other than ADO or coils are needed. In six procedures, we used AVP II, Amplatzer Muscular VSD Occluder or Nit-Occlud PDA-R. AVP II was developed for vascular occlusion in general, not PDA specifically, with two lateral discs and a central component of the same diameter, and has proven valuable to close large PDA of type C, D or E [20]. Amplatzer Muscular VSD Occluder, a double-disc nitinol plug devised for VSD closure, has been described for PDA closure in large PDA associated with pulmonary hypertension [21]. In our patient receiving an Amplatzer Muscular VSD Occluder, closure with ADO I was attempted first, which was unstable as the PDA was characterized by a large aortic ampulla. Nit-Occlud PDA-R is a flexible device consisting of nitinol wire with the ability to adapt to different PDA morphologies [22]. Due to the more complex procedure, longer procedure and fluoroscopy times were needed, but compared to a recent multicenter registry, rate of $\mathrm{AE}$ was low and success rate high in our patients treated with these devices [23].

Complete PDA closure was observed in $61.1 \%$ of patients at the end of catheterization but finally achieved in $98.4 \%$ after median follow-up of 3.3 years. A meta-analysis containing a total of 1808 patients determined a closure rate of $94.0 \%$ at last follow-up, with follow-up time varying from 1 day to 5 years [24]. Studies have shown that residual shunts after transcatheter PDA closure might lead to intravascular hemolysis or endarteritis [25], both of which were not observed in our cohort.

The total rate of AE regardless of severity level appears high (17.2\%), but is similar to a comparable study showing complication rates of $21.5 \%$ in transcatheter PDA closure between 2000 and 2004, with decreasing numbers due to growing technical experience [26]. In our cohort, most AE were of low severity, but in 13 patients $(3.9 \%)$, secondary intervention $(3.3 \%)$ and/or surgery $(0.9 \%)$ was needed.

Intra- or postprocedural device embolization occurred in both most frequently used device groups, in coils (2.7\%) and ADO (2.2\%), with comparable embolization rates described by others of $1.5-9 \%$ for coils and $0-4 \%$ for ADO [27]. Short window type PDA constitutes a predisposition for device embolization [28].

In one 5-month-old infant treated with Cook detachable coil, temporary postprocedural left-sided recurrent laryngeal nerve paralysis occurred. This is rare but described after surgical as well as transcatheter PDA closure, more often after use of coils compared to other devices [29].

Younger age at intervention is a known risk factor for $\mathrm{AE}$, which were more frequent in infants under one year of age (39.6\% vs. 12.5\%). This includes a higher rate of vascular access-related injuries found in one 
publication (8.2\%) [30], coinciding with our rate of vascular access-related AE of 7.5\% in infants. Efforts to minimize access-related complications especially in preterm and low-birthweight infants include ultrasoundguided vessel puncture, use of smaller introducer sheaths and solely transvenous approach [2,31].

The negative correlation found between age at intervention and minimal PDA diameter indicates earlier time of intervention in patients with larger PDA. Our treatment approach is awaiting spontaneous PDA closure until 1-2 years of age, whereafter transcatheter PDA closure is performed. Hemodynamically significant left-to-right shunt and clinical signs of congestive heart failure may lead to earlier intervention, in accordance with guidelines for the CHD management [10,32].

Changes in AP guidelines published by the American Heart Association in 2007 [4], followed by adaptations regarding recommendations in Switzerland [5], resulted in changing practice of transcatheter PDA closure in our center (Fig. 3). After 2009, the annual rate of PDA closures decreased, closed PDA were larger in size and ADO rather than coils were used. This trend was also shown in the UK/Ireland, with a substantial decline in surgical PDA closure between 2000 and 2016 and a slight decrease of transcatheter procedure during the last two years [33]. This might not only be linked to changes in AP guidelines, but also a more general reluctance whether every PDA needs always be closed, especially the very small "silent" PDA and that in preterm infants prone to spontaneous closure [24,34], where debate is still controversial [35].

In the future, transcatheter PDA closure may further evolve as a less invasive alternative to surgical PDA closure even in preterm infants, due to the development of devices such as the Medtronic Micro Vascular Plug with smaller sheaths and less traumatic aspects [36]. Still, risk-benefit analysis, patient selection and optimal timing for PDA closure are essential.

\section{Study Limitation}

The limitations of this study arise from its retrospective single center design, including non-standardized follow-up intervals. Comparisons of subgroups were limited due to small numbers of patients. In addition, as the study was conducted in a center with many referring smaller hospitals or medical practices, our numbers of transcatheter PDA closure have not been correlated with total numbers of PDA in the population or compared to surgical ligations. The strengths of our study are long-term follow-up, description of different device types and overview of intraprocedural solutions dealing with AE as well as the time period of changes in AP guidelines.

\section{Conclusion}

Transcatheter PDA closure is an efficient and safe procedure with high complete closure rate during long-term follow-up (98.4\%) and low relevant complication rate with need for secondary catheter reintervention (3.3\%) or surgical treatment $(0.9 \%)$. Changes in AP guidelines in 2009 changed the daily care practice of PDA closure.

Acknowledgement: We thank the collaborating pediatric cardiologists providing long-term follow-up data, Silvia Hilfiker for preparing data sets and ethical agreement and Professor Felix Berger for performing transcatheter PDA closures during the first years of the study period. Furthermore, we thank patients and families for research consent.

Funding Statement: The authors received no specific funding for this study.

Conflicts of Interest: The authors declare that they have no conflicts of interest to report regarding the present study. 


\section{References}

1. Sathanandam, S., Balduf, K., Chilakala, S., Washington, K., Allen, K. et al. (2019). Role of transcatheter patent ductus arteriosus closure in extremely low birth weight infants. Catheterization and Cardiovascular Interventions, 93(1), 89-96.

2. Baruteau, A. E., Hascoët, S., Baruteau, J., Boudjemline, Y., Angel, C. Y. et al. (2014). Transcatheter closure of patent ductus arteriosus: Past, present and future. Archives of Cardiovascular Diseases, 107(2), 122-132.

3. Callegari, A., Burkhardt, B., Relly, C., Knirsch, W., Christmann, M. (2019). Ductus arteriosus-associated infective endarteritis: Lessons from the past, future perspective. Congenital Heart Disease, 14(4), 671-677.

4. Wilson, W., Taubert, K., Gewitz, M., Lockhart, P., Baddour, L. et al. (2007). Prevention of infective endocarditis. Circulation, 116(15), 1736-1754.

5. Knirsch, W., Günthard, J. (2009). Neue Empfehlungen zur antibiotischen Endokarditisprophylaxe bei Kindern in der Schweiz. Paediatrica, 20(4), 28-34.

6. Elder, R. W., Baltimore, R. S. (2015). The changing epidemiology of pediatric endocarditis. Infectious Disease Clinics of North America, 29(3), 513-524.

7. Krichenko, A., Benson, L. N., Burrows, P., Möes, C. A. F., McLaughlin, P. et al. (1989). Angiographic classification of the isolated, persistently patent ductus arteriosus and implications for percutaneous catheter occlusion. American Journal of Cardiology, 63(12), 877-880.

8. Bergersen, L., Giroud, J. M., Jacobs, J. P., Franklin, R. C. G., Béland, M. J. et al. (2011). Report from The International Society for Nomenclature of Paediatric and Congenital Heart Disease: Cardiovascular catheterisation for congenital and paediatric cardiac disease (Part 2-Nomenclature of complications associated with interventional cardiology), Cardiology in the Young, 21(3), 260-265.

9. Porstmann, W., Wierny, L., Warnke, H. (1967). Closure of persistent ductus arteriosus without thoracotomy. German Medical Monthly, 12(6), 259-261.

10. Lindinger, A., Kozlik-Feldmann, R., Cesnjevar, R. (2017). Persistent arterial duct (PDA). Cardiology in the Young, 27(Suppl. 3), 13-14.

11. Adams, P. E., Chersich, M. F., Cilliers, A. (2018). Transcatheter closure of the patent ductus arteriosus at a public sector hospital in Soweto, South Africa: A review of patient outcomes over 15 years. Cardiovascular Journal of Africa, 29(4), 246-251.

12. O'Byrne, M. L., Kennedy, K. F., Rome, J. J., Glatz, A. C. (2018). Variation in practice patterns in device closure of atrial septal defects and patent ductus arteriosus: An analysis of data from the IMproving Pediatric and Adult Congenital Treatment (IMPACT) registry. American Heart Journal, 196, 119-130.

13. Philip, R., Rush Waller III, B., Agrawal, V., Wright, D., Arevalo, A. et al. (2016). Morphologic characterization of the patent ductus arteriosus in the premature infant and the choice of transcatheter occlusion device. Catheterization and Cardiovascular Interventions, 87(2), 310-317.

14. Masura, J., Walsh, K. P., Thanopoulous, B., Chan, C., Bass, J. et al. (1998). Catheter closure of moderate- to largesized patent ductus arteriosus using the New Amplatzer Duct Occluder: Immediate and short-term results. Journal of the American College of Cardiology, 31(4), 878-882.

15. Thanopoulos, B., Eleftherakis, N., Tzannos, K., Stefanadis, C. (2008). Transcatheter closure of the patent ductus arteriosus using the new Amplatzer duct occluder: Initial clinical applications in children. American Heart Journal, 156(5), 917.e1-917.e6.

16. Agnoletti, G., Marini, D., Villar, A. M., Bordese, R., Gabbarini, F. (2012). Closure of the patent ductus arteriosus with the new duct occluder II additional sizes device. Catheterization and Cardiovascular Interventions, 79(7), $1169-1174$.

17. Baspinar, O., Irdem, A., Sivasli, E., Sahin, D., Kilinc, M. (2012). Comparison of the efficacy of different-sized Amplatzer duct occluders (I, II, and II AS) in children weighing less than $10 \mathrm{~kg}$. Pediatric Cardiology, 34, 88-94.

18. Saliba, Z., El-Rassi, I., Helou, D., Chehab, G., Abdel-Massih, T. et al. (2012). Analyzing the failures of percutaneous closure of the patent ductus arteriosus in patients over $5 \mathrm{~kg}$. Journal of Invasive Cardiology, 24(9), 434-438. 
19. Atiq, M., Aslam, N., Kazmi, K. A. (2007). Transcatheter closure of small-to-large patent ductus arteriosus with different devices: Queries and challenges. Journal of Invasive Cardiology, 19(7), 295-298.

20. Garay, F. J., Aguirre, D., Cárdenas, L., Springmüller, D., Heusser, F. (2015). Use of the Amplatzer vascular plug II device to occlude different types of patent ductus arteriosus in pediatric patients. Journal of Interventional Cardiology, 28(2), 198-204.

21. Thanopoulos, B. D., Tsaousis, G. S., Djukic, M., Al Hakim, F., Eleftherakis, N. G. et al. (2002). Transcatheter closure of high pulmonary artery pressure persistent ductus arteriosus with the Amplatzer muscular ventricular septal defect occluder. Heart, 87(3), 260-263.

22. Heath, A., Lang, N., Levi, D. S., Granja, M., Villanueva, J. et al. (2012). Transcatheter closure of large patent ductus arteriosus at high altitude with a novel nitinol device. Catheterization and Cardiovascular Interventions, 79(3), 399-407.

23. Brunetti, M. A., Ringel, R., Owada, C., Coulson, J., Jennings, J. M. et al. (2010). Percutaneous closure of patent ductus arteriosus: A multiinstitutional registry comparing multiple devices. Catheterization and Cardiovascular Interventions, 76(5), 696-702.

24. Fortescue, E. B., Lock, J. E., Galvin, T., McElhinney, D. B. (2010). To close or not to close: The very small patent ductus arteriosus. Congenital Heart Disease, 5(4), 354-365.

25. Jang, G. Y., Son, C. S., Lee, J. W., Lee, J. Y., Kim, S. J. (2007). Complications after transcatheter closure of patent ductus arteriosus. Journal of Korean Medical Science, 22(3), 484-490.

26. Azhar, A. S., Abd El-Azim, A. A., Habib, H. S. (2009). Transcatheter closure of patent ductus arteriosus: Evaluating the effect of the learning curve on the outcome. Annals of Pediatric Cardiology, 2(1), 36-40.

27. Yarrabolu, T. R., Rao, P. S. (2012). Transcatheter closure of patent ductus arteriosus. Pediatrics \& Therapeutics, S5, 1-8.

28. Ewert, P. (2005). Challenges encountered during closure of patent ductus arteriosus. Pediatric Cardiology, 26(3), 224-229.

29. Liang, C. D., Ko, S. F., Huang, S. C., Huang, C. F., Niu, C. K. (2003). Vocal cord paralysis after transcatheter coil embolization of patent ductus arteriosus. American Heart Journal, 146(2), 367-371.

30. Backes, C. H., Rivera, B. K., Bridge, J. A., Armstrong, A. K., Boe, B. A. et al. (2017). Percutaneous patent ductus arteriosus (PDA) closure during infancy: A meta-analysis. Pediatrics, 139(2), e20162927.

31. Liu, J., Gao, L., Tan, H. L., Zheng, Q. H., Liu, L. et al. (2018). Transcatheter closure through single venous approach for young children with patent ductus arteriosus: A retrospective study of 686 cases. Medicine, 97(35), e11958.

32. Baumgartner, H., Bonhoeffer, P., de Groot, N. M. S., de Haan, F., Deanfield, J. E. et al. (2010). ESC Guidelines for the management of grown-up congenital heart disease (new version 2010): The task force on the management of grown-up congenital heart disease of the European Society of Cardiology (ESC). European Heart Journal, 31(23), 2915-2957.

33. Farooqi, M., Stickley, J., Dhillon, R., Barron, D. J., Stumper, O. et al. (2019). Trends in surgical and catheter interventions for isolated congenital shunt lesions in the UK and Ireland. Heart, 105(14), 1103-1108.

34. Lee, J. A. (2019). Practice for preterm patent ductus arteriosus; focusing on the hemodynamic significance and the impact on the neonatal outcomes. Korean Journal of Pediatrics, 62(7), 245-251.

35. Mitchell, C. C., Rivera, B. K., Cooper, J. N., Smith, C. V., Berman, D. P. et al. (2019). Percutaneous closure of the patent ductus arteriosus: Opportunities moving forward. Congenital Heart Disease, 14(1), 95-99.

36. Sathanandam, S., Justino, H., Rush Waller III, B., Radtke, W., Qureshi, A. M. et al. (2017). Initial clinical experience with the Medtronic Micro Vascular PlugTM in transcatheter occlusion of PDAs in extremely premature infants. Catheterization and Cardiovascular Interventions, 89(6), 1051-1058. 